\title{
A micromechanical model for kink-band formation: Part I - experimental study and numerical modelling
}

\author{
S. Pimenta * , R. Gutkin, S. T. Pinho, P. Robinson \\ The Composites Centre, Department of Aeronautics, South Kensington Campus, \\ Imperial College London. London SW7 2AZ, United Kingdom
}

\begin{abstract}
The initiation and propagation of kink-bands are investigated through an experimental study and numerical modelling. Based on the results achieved, the sequence of events and key features for kink-band formation are identified; particularly, matrix yielding is found to play a crucial role in the process, and fibres are found to fail in the compressive side first. The findings from both the experimental and numerical programmes show a remarkable agreement, and are further applied to the development of an analytical model (Part II of this paper) for kink-band formation.
\end{abstract}

Key words: B. Kinking, D. Mechanical testing, C. Finite elements analysis, C. Micro-mechanics, A. Carbon fibres

\section{Introduction}

Kink-band formation (figure 1.a) is the most common failure mode of fibre reinforced polymer (FRP) composites under longitudinal compression [2]. This paper presents experimental and numerical investigations that were conducted to improve the knowledge on the sequence of events and the key features leading to fibre kinking. The outcome of these studies is used in Part II of this paper [3] to develop an analytical model for kink-band formation.

\footnotetext{
* Corresponding author.

Email address: soraia.pimenta@imperial.ac.uk (S. Pimenta).
}

Pimenta S, Gutkin R, Pinho ST, Robinson P (2009). A micromechanical model for kink-band formation: Part I - Experimental study and numerical modelling. Composites Science and Technology 69, 948-955. DOI $: 10.1016 / j$. compscitech. 2009.02.002 


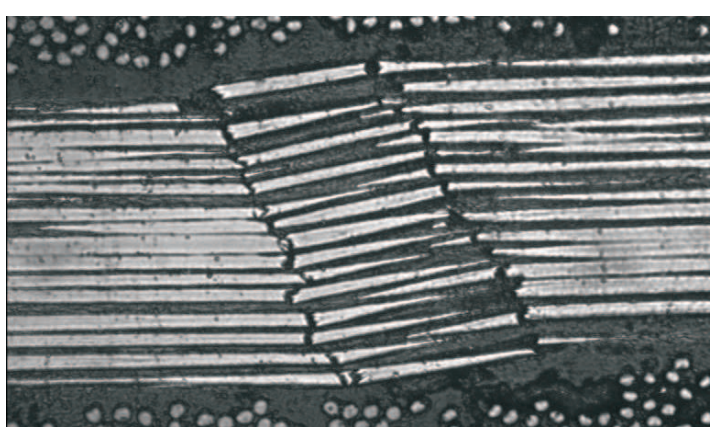

(a) Micrograph from experiments in CFRP [1]

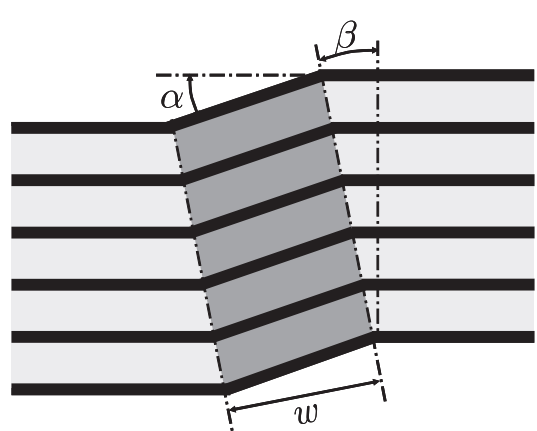

(b) Schematics and definition of the kink-band geometric parameters: fibre angle $\alpha$, band angle $\beta$, and band width $w$.

Fig. 1. Kink-band.

Although there is a large diversity of analytical models aiming to explain and reproduce kink-band formation, no single model has achieved an universal acceptance among researchers and designers [2]. There is therefore the need for a better understanding of the physics and mechanics of fibre kinking, which can be achieved by experimental studies and numerical modelling at the micromechanical scale.

Much information obtained from experimental results on kink-band formation is already available [4-7]. However, kinking is often studied in post-mortem specimens or with low resolution systems, so there is barely no data on the material behaviour at the micromechanical level. The observation of stable and in-plane kink-bands, in loaded specimens and using in-situ microscopy, is therefore required.

Soutis and Fleck [4] tested carbon-epoxy UD composites under uniaxial compression, using both unnotched and open-hole specimens. The main failure mode observed was fibre microbuckling in the 0 plies.

Kyriakydes [5] studied kink-bands through several experimental procedures. Regarding fibre imperfections in a UD composite, the researcher found regularlyspaced bands of fibres with high waviness; the bands were around $1 \mathrm{~mm}$ wide, spaced by $1-1.5 \mathrm{~mm}$. Inside each band, the fibres (diameter $\phi_{f}=7 \mu \mathrm{m}$ ) were randomly misaligned, with half-wavelength and half-amplitude ranging respectively $L \in[1050 ; 2800] \mu \mathrm{m}$ and $\overline{y_{0}} \in[21 ; 70] \mu \mathrm{m}$; no correlation between wavelength and amplitude was found. Kyriakydes suggested these bands to be caused by tow superposition during ply manufacture.

Moran [6] has observed almost in-plane kink-bands by compressing thick $(6 \mathrm{~mm})$ UD notched specimens. The author then proposed the following sequence of events for the phenomenon: (i) initially linear-elastic behaviour; (ii) matrix yielding around the notch; (iii) peak load and sudden kink-band propagation across the entire specimen width; (iv) slow increase of fibre rotation 
$\alpha$ within the band; (v) unstable increase of fibre rotation $\alpha$ and band angle $\beta$ until lock-up by matrix response in shear; (vi) band broadening.

Vogler and Kyriakydes [7] achieved a stable and almost in-plane propagation of kink-bands in UD CFRP, using compression combined with shear. Unloaded micrographs showed almost no fibre failure, due to a relatively small fibre angle $\alpha$. Their results suggest that shear stresses play a significant role in fibre kinking, even leading to the formation of kink-bands without fibre failure. In addition, and despite the effort to produce totally in-plane kink-bands, an out-of-plane component was observed in loaded specimens.

Several micromechanical FE models were also developed to simulate the composite behaviour during kink-band formation, considering matrix yielding and initial imperfections $[5,8,9]$. However, there are no studies reported on kinking in initially perfect fibres and using a failing formulation for the matrix.

Kyriakides et al [5] performed an extended study on the influence of several parameters on the composite response and kink-band geometry. The FE models used a 2D layered - fibres interposed with matrix - approximation. The matrix was formulated as elasto-plastic; the fibres were considered isotropic, and their material non-linearity had only a reduced influence on the results. All the models assumed a sinusoidal initial imperfection. In the overall, the simulations showed (i) an initial linear-elastic domain, (ii) a peak load, (iii) a sudden softening with unstable kink-band formation, and (iv) a stable band broadening. It was also found that the addition of more fibres to the model would increase the peak remote stress.

Morais [8] used a unit-cell numerical approach. In his 3D models this author found that, if a hexagonal arrangement for the fibres is considered, kinking is isotropic. For this reason, this type of fibre arrangement will be assumed in the models presented in this paper.

Vogler et al. [9] modelled kink-band propagation in FE analyses, using both global and local imperfections and a plastic formulation for the matrix. The numerical simulations reproduced the behaviour previously observed in the experiments [7]; it was shown that a much more stable numerical response was achieved if direct shear loading was introduced.

The experimental and numerical programmes presented in this paper aim to study the formation of kink-bands at the micro-scale. The final goal is to provide useful information on the physics and micromechanics of the process, so a phenomenological analytical model can be developed in Part II of this paper [3]. This paper is organised as follows: sections 2 and 3 present respectively the experimental and numerical procedures carried out; based on the results obtained, the sequence of events leading to kink-band formation is proposed in section 4, and key features for kinking are identified in section 5; section 6 


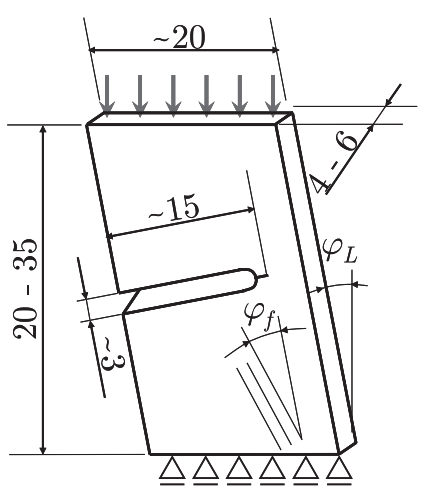

Fig. 2. Specimen used in the experimental study and imposed displacements. All dimensions in $\mathrm{mm}$.

summarizes the main conclusions.

\section{Experimental study}

The material used in the experiments is a T800/924 carbon-epoxy unidirectional (UD) pre-preg. Small and thick UD specimens [6] (figure 2) were machined from plates manufactured using pre-preg technology by the standard procedures. A long notch was made with a band saw and sharpened with a modelling blade to assist kink-band initiation. The off-axis scheme shown in figure 2 , with angles $\varphi_{f}$ or $\varphi_{L}$ under $5^{\circ}$, was used to induce shear and in-plane kink-band propagation $[7,9]$.

Kink-band initiation was achieved by compressing each specimen in a vice, after which the specimen was reloaded in a small portable clamp; propagation was observed while loaded and unloaded under optical and scanning electron microscopes. Sample micrographs - taken at a considerable distance from the notch, so stress concentration can be neglected - are shown in figures 3, 4 and 5 .

\section{$3 \quad$ Numerical modelling}

Kink-band formation was simulated using several 2D equivalent $[5,8,9]$ micromechanical FE models of the composite. The overall approach consisted of compressing a large number of fibres with free longitudinal boundaries, as performed by Kyriakydes and Vogler [5,9]; this allows the kink-band angle $\beta$ to be different from 0 , keeping at the same time a considerable number of inner fibres sufficiently distant from free-edge effects. The thicknesses of the fibre and matrix layers were derived using the approach suggested by Morais, 


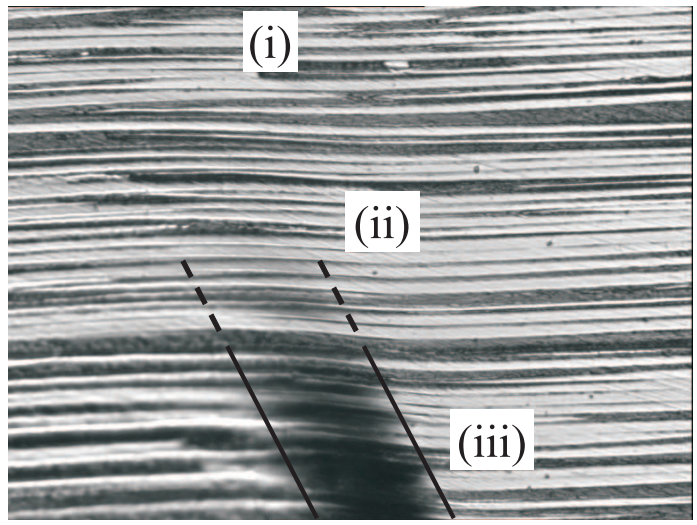

(i) elastic domain; (ii) softening domain; (iii) fibre failure domain.

Fig. 3. Stages of fibre kinking (loaded).

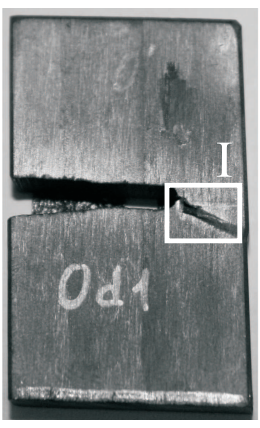

(a) Overview.

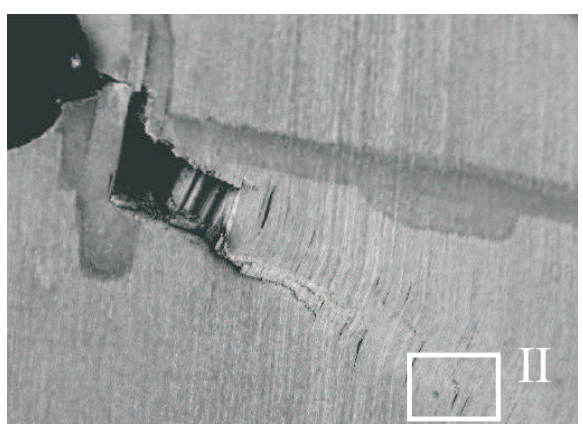

(b) Zoom-in of zone I.

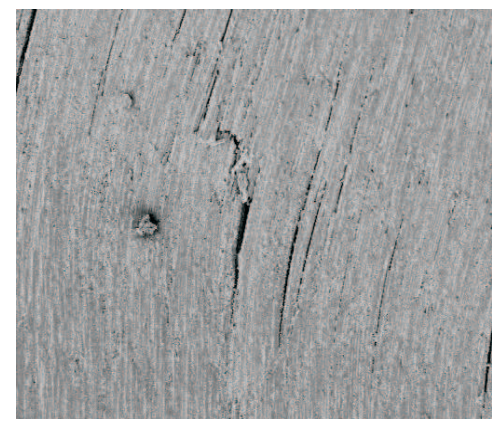

(c) Zoom-in of zone II.

Fig. 4. Kink-band with matrix yielding and no fibre failure (unloaded).

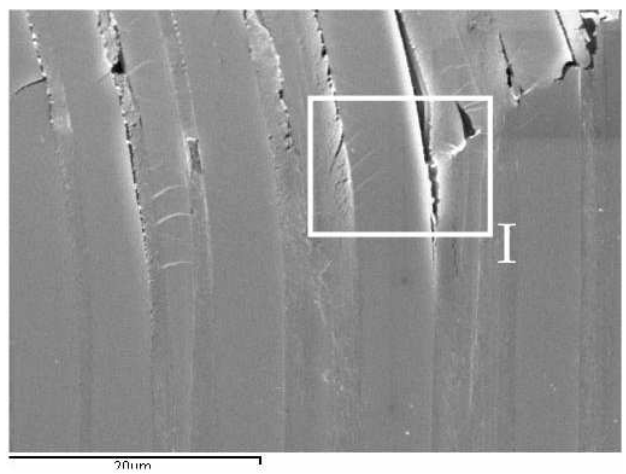

(a) Overview of kink-band edge.

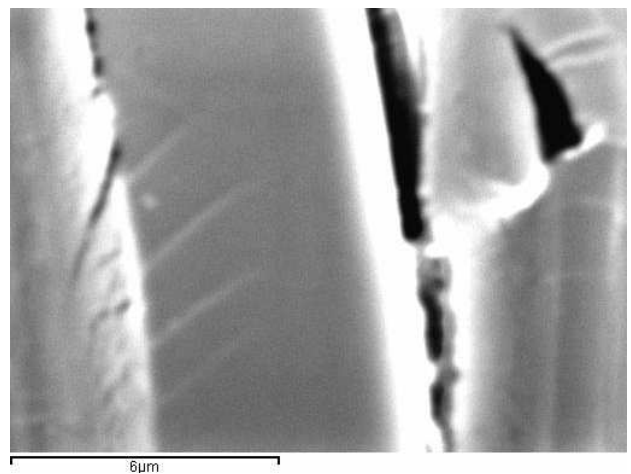

(b) Zoom-in of zone I.

Fig. 5. Detail of kink-band edge and fibre failure in compression (loaded) [12].

considering a symmetry plane in an hexagonal fibre arrangement [8]. The main parameters that were analysed with the numerical simulations include:

- matrix failure: models with matrix yielding (plasticity model) and with matrix failure (cohesive model) were investigated;

- the length of the fibres in the model; 


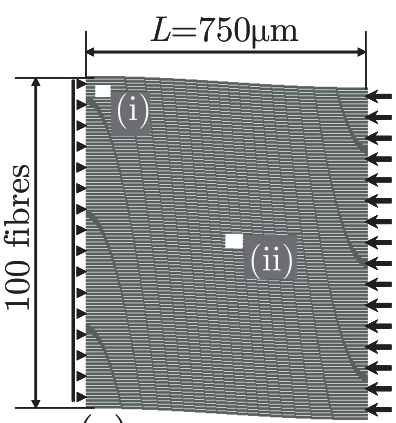

(a)
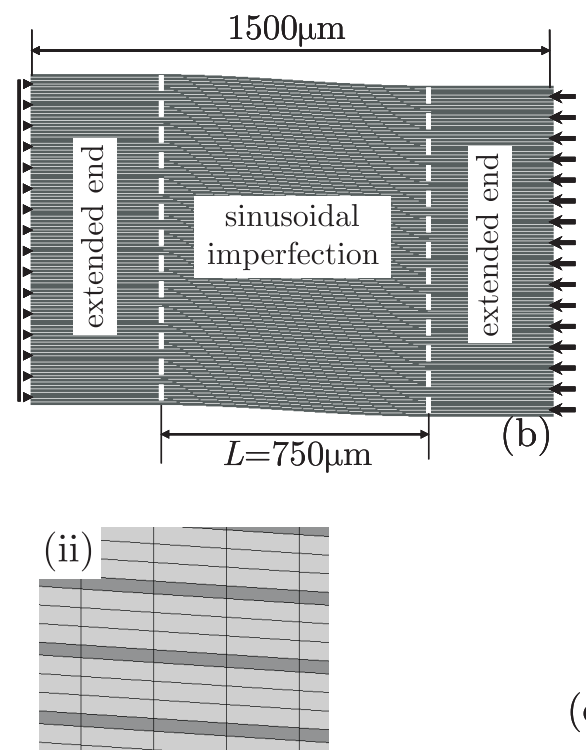

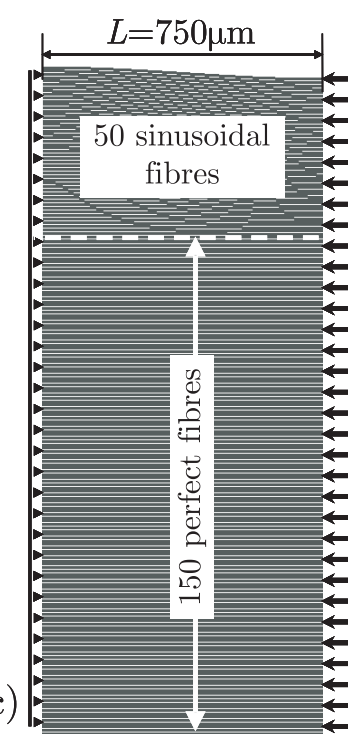

(a) model containing fibres with initial geometrical imperfection; magnified details of the mesh are shown in (a.i) and (a.ii);

(b) model with fibres with extended straight segments;

(c) model containing both sinusoidal (with the amplitude decreasing linearly with the fibre index) and straight (perfect) fibres.

Fig. 6. Geometries, meshes and boundary conditions of the numerical models.

- the initial shape of the fibres (sinusoidal and straight);

- fibre failure: models with purely elastic fibres and with fibres which are allowed to fail (using a continuum damage mechanics (CDM) model) were investigated.

The main conclusions from the study can be drawn by analysing the results corresponding to three different models (see also figure 6):

- Cohesive model, with elastic fibres, cohesive matrix (failing bi-linear constitutive law, modelled with cohesive elements) and global imperfection (figure 6.a);

- $C D M_{f}$ model, with failing (bi-linear constitutive law) fibres, elasto-plastic matrix and global imperfection (figure 6.b). This model has extended ends, so the kink-band can be studied at later stages - when considerable fibre failure is verified - without being affect by edge effects;

- Propagation model, with elastic fibres, cohesive matrix and both initially misaligned and straight fibres (figure 6.c).

The analysis were run in the finite element code Abaqus [10], by the static solver and using geometric non-linearity. Numerical stabilization was added to the models here presented, by specifying a constant damping factor (5000 for the cohesive and propagation models, 500 for the $C D M_{f}$ model). The artificial energy dissipated by damping was monitored throughout the analyses, and always found to be less than $10 \%$ of the total energy; in addition, models run with no stabilization showed a very similar response (apart from increased 


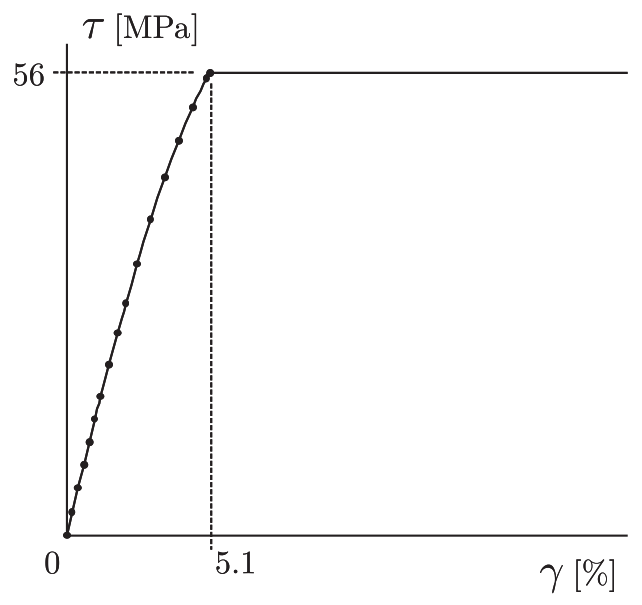

Fig. 7. Non-linear elastic-plastic constitutive law for the matrix $\left(C D M_{f}\right.$ model $)$.

convergence problems).

Plane-strain elements with reduced integration were used for the fibres (all models) and for the matrix with elasto-plastic formulation $\left(C D M_{f}\right.$ model). Cohesive (failing) matrix (cohesive and propagation models) was modelled using Abaqus's cohesive elements, and fibre failure $\left(C D M_{f}\right.$ model) using the Continuous Damage Mechanics (CDM) model for FRP (with transverse modes deactivated); bi-linear laws were used in both cases, but to avoid convergence problems matrix failure and fibre failure were studied in independent models. An experimental [11] shear curve was used for the elasto-plastic matrix $\left(C D M_{f}\right.$ model) up to a shear strain of $5.1 \%$ (limit of experimental data), with a perfect-plastic response for larger deformations (figure 7).

Kink-band formation was triggered by a sinusoidal imperfection. The amplitude of this imperfection was constant for all the fibres in the cohesive and $C D M_{f}$ models (figure 6.a-b); in the propagation model, the nominal (for the first fibre) amplitude was linearly reduced to zero along the following 50 fibres (figure 6.c). For the three models presented, the nominal imperfection (table 4) was $30 \%$ proportionally smaller than the range measured by Kyriakydes [5]; no significant qualitative difference was found in models with different amplitude parameters.

The models were loaded in longitudinal compression with displacement control. The geometry, mesh and boundary conditions are shown in figure 6 . Tables 1 to 4 present the input parameters used in the model $[1,11]$. Results are shown in figures 8, 9, 10 and 11 . 
Table 1

Constituents elastic properties used in the numerical models (typical values for IM7/8551-7 from [11]).

\begin{tabular}{cccc}
\hline \hline Fibre & Fibre & Matrix & Matrix \\
Young's Modulus & $\begin{array}{c}\text { Poisson's ratio } \\
E_{f}[\mathrm{MPa}]\end{array}$ & $\begin{array}{c}\text { Shear Modulus } \\
\boldsymbol{G}_{m}[\mathrm{MPa}]\end{array}$ & $\begin{array}{c}\text { Poisson's ratio } \\
\nu_{m}[]\end{array}$ \\
\hline 276 & 0.20 & 1.478 & 0.38 \\
\hline \hline
\end{tabular}

Table 2

Constituents strength properties used in the numerical models (typical values for IM7/8551-7 from [11]).

\begin{tabular}{cccc}
\hline \hline $\begin{array}{c}\text { Fibre Compression } \\
X_{C}^{f}[\mathrm{MPa}]\end{array}$ & $\begin{array}{c}\text { Fibre Tension } \\
X_{T}^{f}[\mathrm{MPa}]\end{array}$ & $\begin{array}{c}\text { Matrix Mode I } \\
Y_{m}[\mathrm{MPa}]\end{array}$ & $\begin{array}{c}\text { Matrix Shear } \\
S_{m}[\mathrm{MPa}]\end{array}$ \\
\hline 3200 & 5180 & 73 & 56 \\
\hline \hline
\end{tabular}

Table 3

Constituents fracture toughness used in the numerical models (values based on [1]).

\begin{tabular}{cccc}
\hline \hline $\begin{array}{c}\text { Fibre Compression } \\
\mathcal{G}_{C}^{f}\left[\mathrm{~kJ} / \mathrm{m}^{2}\right]\end{array}$ & $\begin{array}{c}\text { Fibre Tension } \\
\mathcal{G}_{T}^{f}\left[\mathrm{~kJ} / \mathrm{m}^{2}\right]\end{array}$ & $\begin{array}{c}\text { Matrix Mode I } \\
\mathcal{G}_{I C}^{m}\left[\mathrm{~kJ} / \mathrm{m}^{2}\right]\end{array}$ & $\begin{array}{c}\text { Matrix Mode II } \\
\mathcal{G}_{I I C}^{m}\left[\mathrm{~kJ} / \mathrm{m}^{2}\right]\end{array}$ \\
\hline 100 & 100 & 0.21 & 0.80 \\
\hline \hline
\end{tabular}

Table 4

Geometrical and numerical composite parameters used in the numerical models.

\begin{tabular}{ccccc}
\hline \hline Imperfection & Imperfection & Fibre & Fibre \\
$1 / 2$ wavelength & $1 / 2$ amplitude \\
$L[\mu \mathrm{m}]$ & $\overline{y_{0}[\mu \mathrm{m}]}$ & $\begin{array}{c}\text { Molume fraction } \\
V_{f}[\%]\end{array}$ & $\begin{array}{c}\text { diameter } \\
\phi_{f}[\mu \mathrm{m}]\end{array}$ & $\begin{array}{c}\text { BK exponent } \\
\eta[]\end{array}$ \\
\hline 750 & 15 & 60 & 7 & 1.5 \\
\hline \hline
\end{tabular}

\section{Sequence of events}

Figure 3 suggests the existence of three domains in kink-band formation. In the elastic domain - region (i), way from the kink-band tip - the fibres show a waviness with small amplitude and high wavelength. In the softening domain - region (ii), just ahead the kink-band tip - the waviness is more pronounced and more localised (higher amplitude and lower wavelength). In the fibre failure domain - region (iii) - a shaded kink-band is observable; the fibres at the band edges are broken, which (together with the free-surface effect) allows the kink-band to acquire a considerable out-of-plane component, which is responsible for the shading in that region.

In figure 8.b, these three domains can also be distinguished: in the softening domain the matrix is progressively degraded; in the fibre failure domain, the 


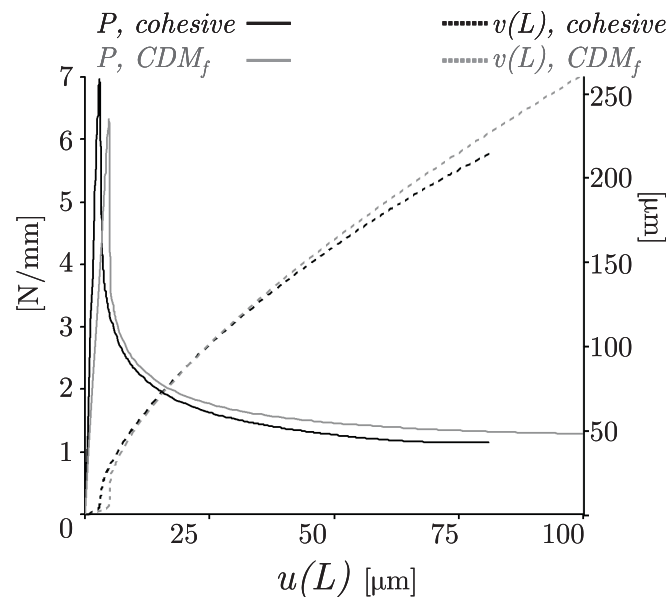

(a) Load $P$ and transverse displacement $v$ vs. compressive displacement $u$.

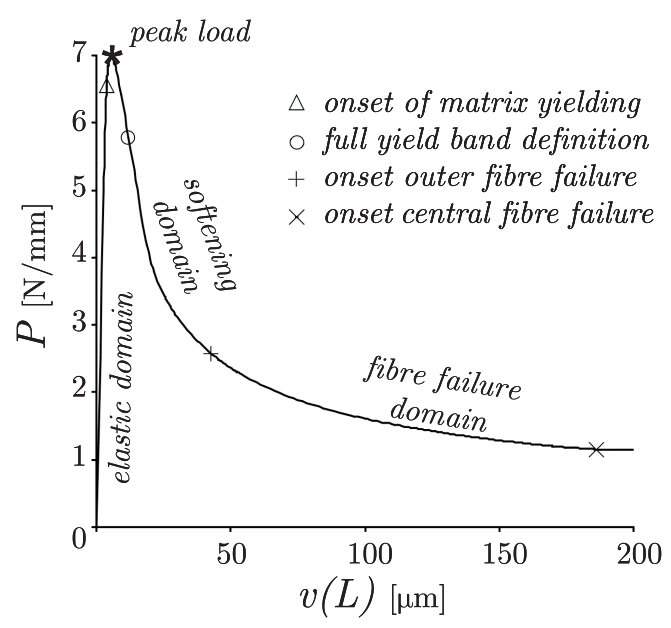

(b) Load $P$ vs. transverse deflection $v(L)$ (cohesive model).

Fig. 8. Load vs. displacement curves from numerical models.

strength of the fibres is reached, starting from the outer fibres and progressing towards the central ones (although, in the cohesive model, no fibre-stiffness degradation results from overcompression). Regarding the sequence in which all these events take place, a macroscopic kink-band where matrix yielding can be observed prior to fibre failure is shown in figure 4.c.

Combining all these results with the fields obtained from the numerical analysis (figure 9), the following sequence of events for kink-band formation is proposed:

1. Elastic domain: fibres and matrix deform elastically; the imperfection induces slight bending of the fibres and shears the matrix, amplifying moderately the misalignment in a positive feedback process (figure 9.a, d, g).

2. Beginning of the softening domain and peak load: as compression continues, the matrix yields in shear within an inclined band developed across all the layers along the full model width (full yield band definition, figure 9.b). The limited support given there to the fibres promotes deflection in a kinked (not sinusoidal) shape (figure 9.e), with highly curved and stressed regions near the yield band boundaries (in the maximum bending bands, figure 9.h). The overall stiffness drops suddenly, so the load $P$ decreases and the transverse displacement $v$ increases abruptly (figure 8.a). In the FE results, due to use of numerical stabilization and to the edge effect (finite number of fibres), the peak load is artificially increased and the transition between the elastic and softening domains is smoothed (figure 8.b).

3. Softening domain (post-peak): after the peak load is reached, fibre rotation increases locally within a widening yield band; at its boundaries, axial bending stresses increase within the maximum bending bands (figure 9.c, f, i). 


\section{II}

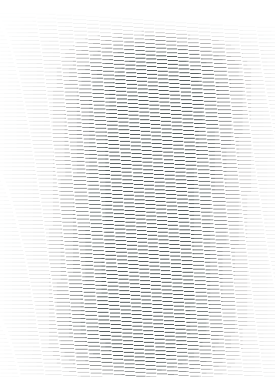

$\tau_{12}^{m} \quad 0$

[MPa]

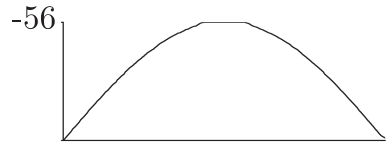

(b) $\tau_{12}^{m} @$ full yield band definition.
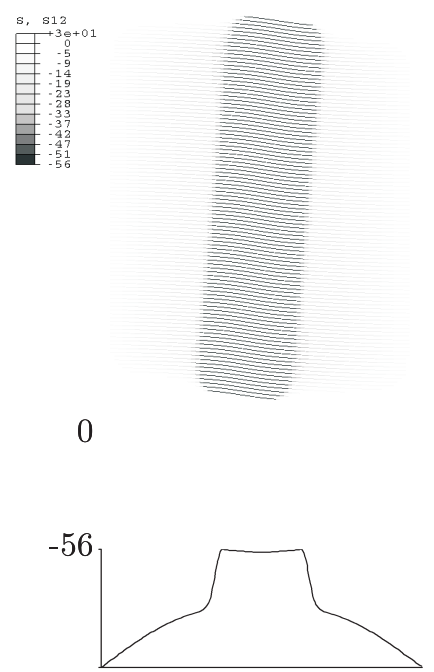

0

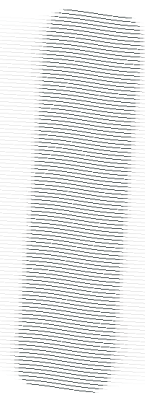

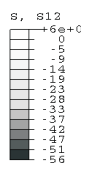

0
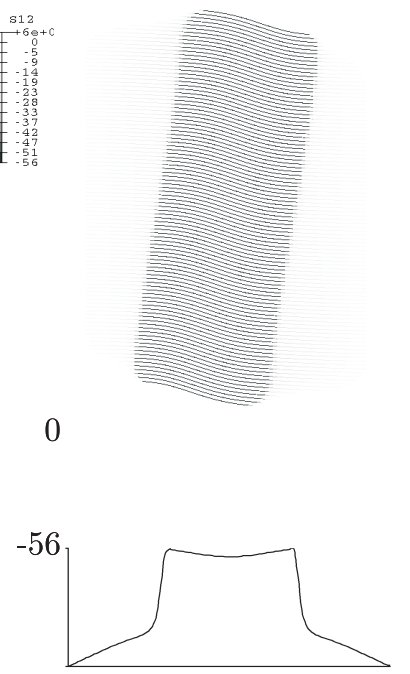

(c) $\tau_{12}^{m} @$ onset outer fibre failure.
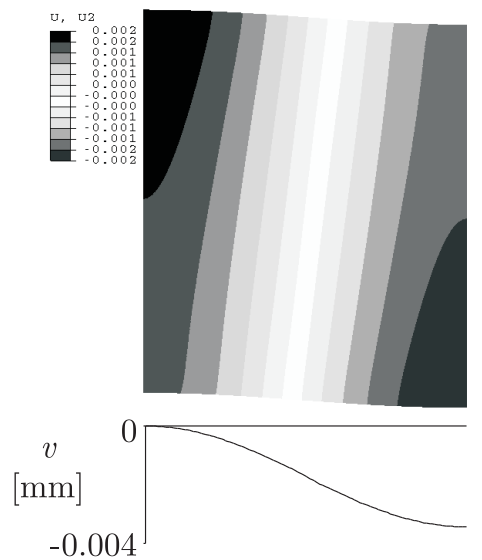

(d) $v @$ onset of matrix yielding.

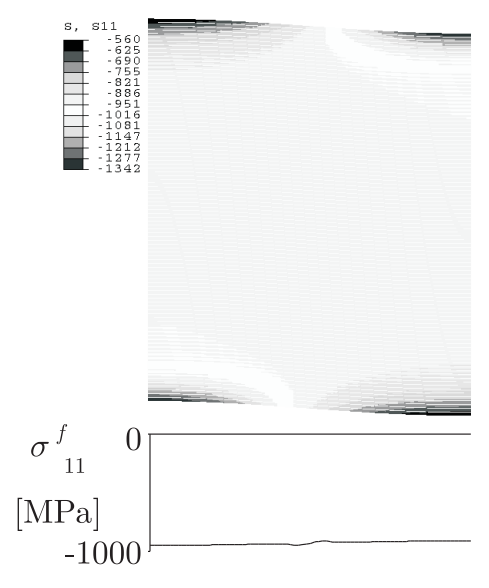

(g) $\sigma_{11}^{f} @$ onset of matrix yielding.
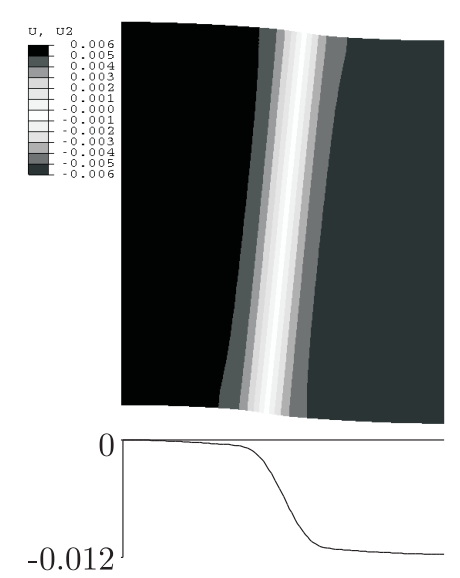

(e) $v @$ full yield band definition.
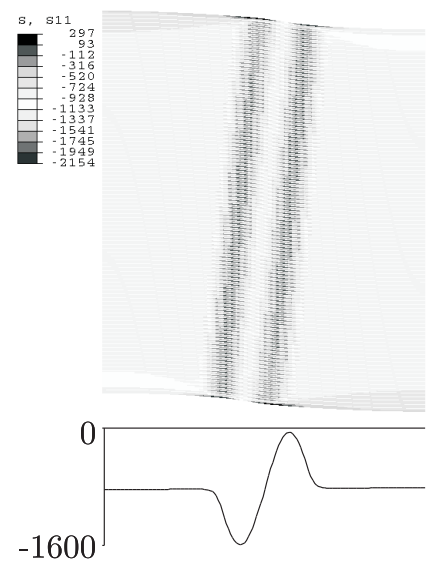

(h) $\sigma_{11}^{f} @$ full yield band definition.

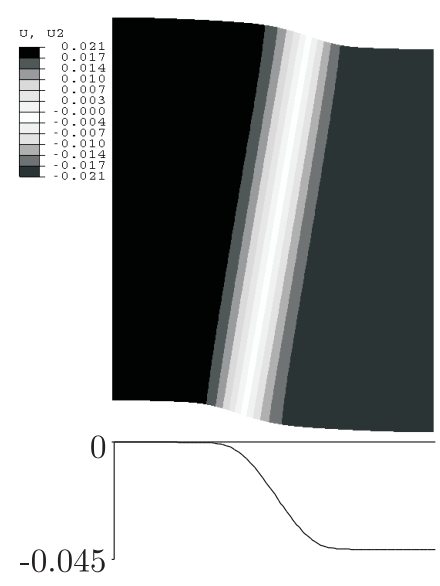

(f) $v$ @ onset outer fibre failure.

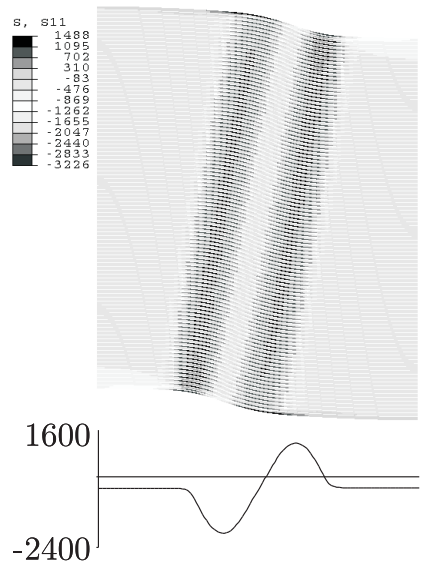

(i) $\sigma_{11}^{f} @$ onset outer fibre failure.

Fig. 9. Fields from numerical analysis (curves for the central fibre and adjoining interface), at the points highlighted in figure 8.b. 


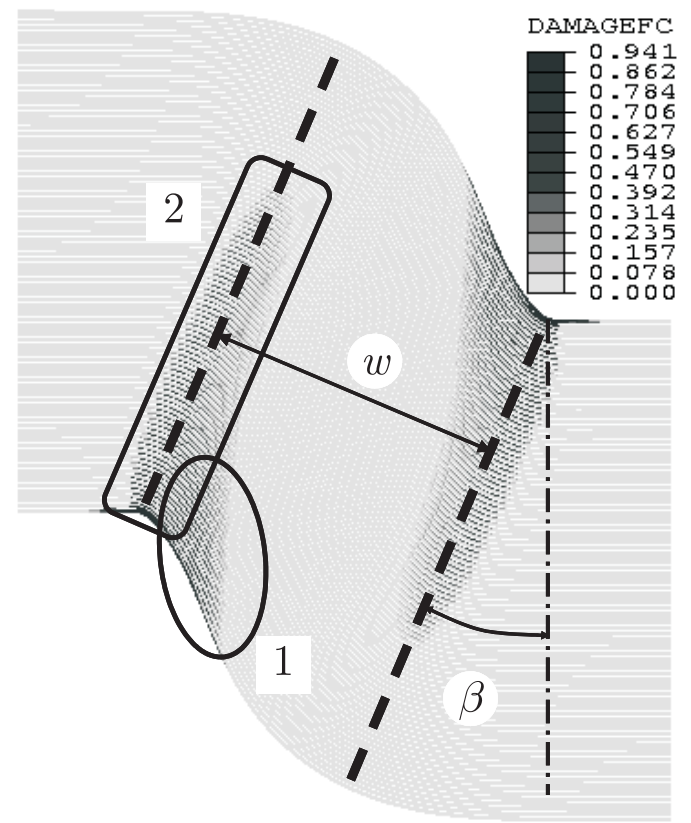

1: Damage propagation along the edge; 2: Damage propagation across the width.

Fig. 10. Fibre failure in compression (index DAMAGEFC) and damage propagation during kink-band formation.

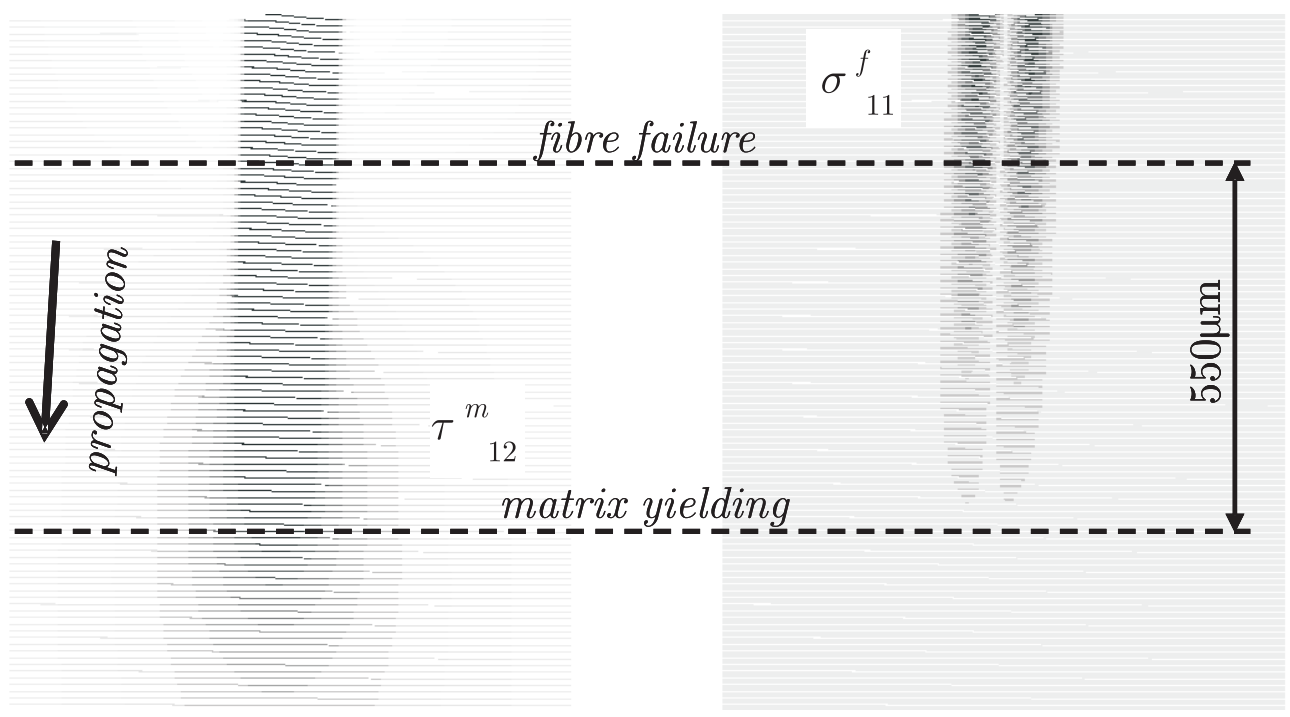

(a) Shear stresses in the matrix.

(b) Axial stresses in the fibres.

Fig. 11. Kink-band propagation in initially straight fibres.

4. Fibre failure domain: as fibre curvature increases in the maximum bending bands, their strength is reached and failure starts at the outer fibres (figures 9.i and 10-feature 1); as the damage propagates inwards (away from edgeeffects), the location and orientation $(\beta)$ of the yield and maximum bending bands stabilizes (and so does $w$, figure 10-feature 2), and only fibre rotation $(\alpha)$ increases until final fibre failure occurs and the kink-band geometry is 


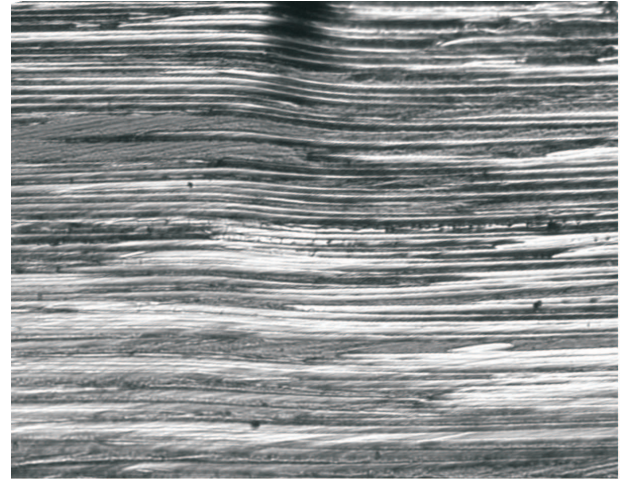

(a) Experimental (loaded) result.

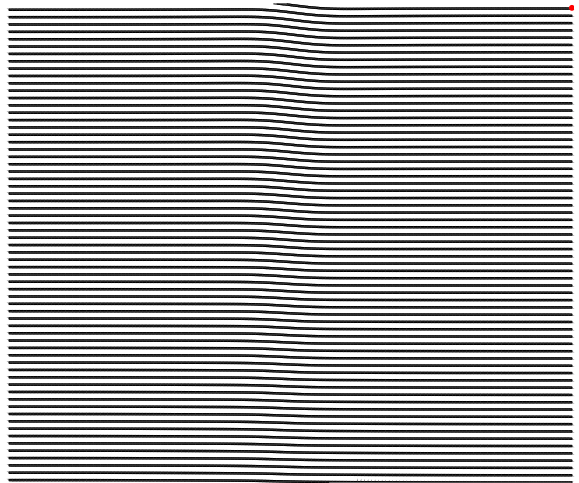

(b) Numerical (propagation) result.

Fig. 12. Kink-band propagation: experimental and numerical results (same scale).

fully defined.

The failure sequence described above is based on the experimental results and on numerical models with global imperfection; in addition, it is also observed in numerical kink-band propagation across initially straight fibres. Figure 11.a shows the matrix shear stresses in a portion of the propagation model with perfect fibres; an inclined yield band propagating downwards is clearly defined in the centre of the model. Figure 11.b shows the axial stresses in the fibres in the same location; the fibres are bent very locally within the yield band, forming two maximum bending bands; the axial stresses in these bands progressively increase, so the compressive fibre strength is reached (no stiffness-reduction due to fibre-overcompression was considered in this model).

It can also be confirmed in figure 11 that, during kink-band propagation, the yield band runs ahead of the maximum bending bands; in the propagation model, matrix yielding occurred over a length approximately $550 \mu \mathrm{m}$ longer than that of failing fibres. This supports that matrix yielding (i) is the responsible for the kinked shape of the fibres, and (ii) takes place prior to onset of fibre failure.

Finally, figure 12.a shows a micrograph from the experimental study of a loaded kink-band propagating, while figure 12.b represents a set of the initiallyperfect fibres in the numerical propagation model. Both figures are at the same scale, and the agreement between experimental and numerical results is remarkable.

\section{$5 \quad$ Key features}

The numerical and experimental results suggest the following features: 
- Two different domains - elastic domain and softening domain — must be considered in kink-band formation (figure 3, features i-ii); matrix yielding is the feature distinguishing them (figures 8 and 9 );

- Fibres deform in bending and compression, and are laterally supported by the matrix in shear (figures 3, 4 and 9);

- For small strains, the fibres deform approximately in-phase (figure 9.a, d, $\mathrm{g})$; however, inside the yield band the out-of-phase angle is not negligible (figure $9 \mathrm{c}, \mathrm{f}, \mathrm{i}$ );

- The similarity between the results from the cohesive and $C D M_{f}$ models (figure 8.a) shows that matrix non-linearity for small strains is not essential, and neither is the choice of modelling the matrix non-linear behaviour for larger strains as plasticity or cohesive failure. This agrees with published work according to which kink-band formation is matrix-strength (and not matrix-toughness) dominated [12];

- Each fibre is deflected over a small length (figures 3 and 12.a);

- Fibres are damaged first in compression; this is supported both by experimental micrographs (figure 5) and by numerical results (figure 10). This finding contrasts with a conclusion attributed to Soutis and Fleck [4] by several other publications $[13,14]$, but not explicited in the original work in reference [4]. This finding (initiation of fibre failure in the compressive side) is however in agreement with other published work [15];

- Matrix yielding in shear is a key feature for kink-band formation (figure 4); fibre failure is simply a consequence of the displacement field after a kink-band (due to matrix failure) is observable (figure 9.d-i).

\section{Conclusions}

Kink-band formation was studied at the micromechanical level in experimental and numerical programmes, and the phenomenological basis for the development of an analytical micromechanical model was established.

The experimental observations were performed with UD CFRP, using notched specimens and off-axis compression. Stable and almost in-plane kink-bands were observed, both while loaded and unloaded, using optical and scanningelectron microscopy. It was shown that kink-band formation does not rely on fibre failure, but on matrix yielding; when fibres do fail, damage starts in compression first.

Kinking was simulated with several numerical FE models. The influence of the matrix behaviour - plastic vs. failing - was studied, and a linear-elastic perfectly-plastic constitutive law proved to be sufficient for an accurate modelling. Fibre failure was simulated, and it was suggested that the kink-band width and orientation are locked after the onset of fibre failure. Propagation 
of kinking was observed in fibres with no initial imperfection, and it was found that considering a small initial sinusoidal imperfection does not affect the mechanics of the process. In addition, and although no quantitative comparison was performed, the numerical results proved to have a remarkable qualitative agreement with the experimental ones.

A sequence of events leading to fibre kinking was proposed. According to this, the composite begins deforming globally in an elastic domain, until matrix yielding initiates and the peak load is reached; then, within the softening domain, the deformation localizes within a narrow band, and the composite softens due to further matrix yielding; finally, in the fibre failure domain, fibres are gradually damaged — starting in the compressive side — until ultimate failure occurs.

Some key features for kink-band formation were also identified; above all, the important role of matrix yielding was confirmed at the micromechanical level. As the matrix yields, the composite softens locally, the deflection increases within a narrow band and, consequently, the fibres may fail near its boundaries. It is matrix yielding - and not any type of local instability or fibre failure - that leads to the definition of the peak load and promotes all the post-peak events - including fibre failure (when occurring) and the definition of kink-band geometry. Another important numerical finding is that the matrix provides lateral support to the fibres until the onset of fibre failure.

The outputs from the experimental and numerical programmes, namely the sequence of events and the key features, are applied to the development of an analytical micromechanical model for kink-band formation, presented in Part II of this paper [3].

\section{Acknowledgments}

The funding from Portuguese Foundation for Science and Technology (project nr. POCTI/EME/50354/2003) and University of Porto (Erasmus Programme) is acknowledged. The Engineering and Physical Sciences Research Council, the UK Ministry of Defence, Airbus and Renault F1 under the project EP/E0Z3169/1 are also gratefully acknowledged. The authors would like to thank Professor P.T. Curtis of the Defence Science and Technology Laboratory (DSTL) for many helpful discussions. 


\section{References}

[1] S.T.Pinho, P.Robinson, L.Iannucci. Fracture toughness of the tensile and compressive fibre failure modes in laminated composites. Composites Science and Technology, Vol. 66 (2006).

[2] C.R.Schultheisz, A.M.Waas. Compressive failure of composites, part I: testing and micromechanical theories. Progress Aerospace Science, Vol. 32 (1996).

[3] S.Pimenta, R.Gutkin, S.T.Pinho, P.Robinson. A micromechanical model for kink-band formation: Part II - analytical modelling. Submitted to Composites Science and Technology (2009).

[4] C.Soutis, N.A.Fleck. Static compression failure of carbon fibre T800/924C composite plate with a single hole. Journal of Composite Materials, Vol. 24 (1990).

[5] S.Kyriakides, R.Arseculeratne, E.J.Perry, K.M.Liechti. On the compressive failure of fiber reinforced composites. International Journal of Solids and Structures, Vol. 32 (1995).

[6] P.M.Moran, X.H.Liu, C.F.Shih. Kink band formation and band broadening in fiber composites under compressive loading. Acta Metallurgica et Materialia, Vol. 43 (1995).

[7] T.J.Vogler, S.Kyriakides. On the initiation and growth of kink bands in fiber composites. Part I: experiments. International Journal of Solids and Structures 38 (2001).

[8] A.B.Morais. Modelling Lamina Longitudinal Compression Strength of Carbon Fibre Composite Laminates. Journal of Composite Materials, Vol. 30 (1996).

[9] T.J.Vogler, S.Y.Hsu, S.Kyriakides. On the initiation and growth of kink bands in fiber composites. Part II: analysis. International Journal of Solids and Structures 38 (2001).

[10] Abaqus, Inc. Abaqus 6.6-1 Documentation. Abaqus. Inc, USA (2006).

[11] M.J.Hinton, P.Soden, A.S.Kaddour. Instructions to contributers of the Second World-Wide Failure Exercise (WWFE-II): Part A. Submitted to Composite Science and Technology (2009).

[12] R.Gutkin, S.T.Pinho, P.Robinson, P.T.Curtis. Physical mechanisms associated with initiation and propagation of kink-bands. In: Proceedings of the 13th European Conference on Composite Materials (ECCM13), Stockholm, Sweden (2008).

[13] N.A.Fleck, L.Deng, D.Budiansky. Prediction of Kink Band Width in Compressed Fiber Composites. Journal of Applied Mechanics, Vol. 62 (1995).

[14] N.A.Fleck. Compressive failure of fiber composites. Advances in Applied Mechanics, Vol. 33 (1997). 
[15] H.T.Hahn, M.Sohi, S.Moon. Compression Failure Mechanisms of Composite Structures. NASA Contractor Report 3988, Langley Research Center (1986).

[16] S.Pimenta. Micromechanics of kink-band formation. Masters thesis, Department of Aeronautics, Imperial College London (2008). 